\title{
Facilitation of Contextual Fear Extinction by Orexin-1 Receptor Antagonism Is Associated with the Activation of Specific Amygdala Cell Subpopulations
}

\author{
África Flores, PhD; Cyril Herry, PhD; Rafael Maldonado, MD, PhD; \\ Fernando Berrendero, $\mathrm{PhD}$
}

\begin{abstract}
Laboratory of Neuropharmacology, Department of Experimental and Health Sciences, Universitat Pompeu Fabra, Barcelona, Spain (Drs Flores, Maldonado, and Berrendero); INSERM, Neurocentre Magendie, Bordeaux, France (Dr Herry); University Bordeaux, Neurocentre Magendie, Bordeaux, France (Dr Herry); Faculty of Experimental Sciences, Universidad Francisco de Vitoria, Madrid, Spain (Dr Berrendero).
\end{abstract}

Correspondence: Rafael Maldonado, MD, PhD, Laboratory of Neuropharmacology, Department of Experimental and Health Sciences, Universitat Pompeu Fabra, PRBB, C/ Doctor Aiguader 88, 08003 Barcelona, Spain (rafael.maldonado@upf.edu); and Fernando Berrendero, PhD, Faculty of Experimental Sciences, Universidad Francisco de Vitoria, 28223 Pozuelo de Alarcón, Madrid, Spain (fernando.berrendero@ufv.es).

\begin{abstract}
Background: Orexins are hypothalamic neuropeptides recently involved in the regulation of emotional memory. The basolateral amygdala, an area orchestrating fear memory processes, appears to be modulated by orexin transmission during fear extinction. However, the neuronal types within the basolateral amygdala involved in this modulation remain to be elucidated.

Methods: We used retrograde tracing combined with immunofluorescence techniques in mice to identify basolateral amygdala projection neurons and cell subpopulations in this brain region influenced by orexin transmission during contextual fear extinction consolidation.

Results: Treatment with the orexin-1 receptor antagonist SB334867 increased the activity of basolateral amygdala neurons projecting to infralimbic medial prefrontal cortex during fear extinction. GABAergic interneurons expressing calbindin, but not parvalbumin, were also activated by orexin-1 receptor antagonism in the basolateral amygdala.

Conclusions: These data identify neuronal circuits and cell populations of the amygdala associated with the facilitation of fear extinction consolidation induced by the orexin-1 receptor antagonist SB334867.
\end{abstract}

Keywords: fear extinction, orexin, amygdala, retrograde labeling, calbindin

\section{Introduction}

Orexins (also known as hypocretins) are hypothalamic neuropeptides that activate 2 different G-protein coupled receptors: orexin type 1 (OX1R) and 2 (OX2R) (de Lecea et al., 1998; Sakurai et al., 1998). Orexin-expressing neurons are exclusively located in the lateral hypothalamus, but they have extensive projections throughout the brain (Peyron et al., 1998). The orexin system is involved in multiple physiological functions (Li et al., 2014), including the modulation of emotional memory (Flores et al., 2015).
Clinical research showed a reduced amygdala activity during aversive conditioning in human narcolepsy (Ponz et al., 2010), a pathophysiological condition associated with a loss of orexin neurons (Peyron et al., 2000). Pharmacological blockade or genetic deletion of OX1R impaired contextual and cued fear conditioning (Sears et al., 2013; Soya et al., 2013; Flores et al., 2014; Wang et al., 2017) in rodents. Moreover, OX1R antagonism facilitated the consolidation of both contextual and cued fear 
extinction (Flores et al., 2014). Interestingly, in the contextual test, the extinction-facilitating effects of the OX1R antagonist SB334867 were associated with increased c-Fos expression in the basolateral amygdala (BLA), suggesting an involvement of this brain area (Flores et al., 2014). Local blockade of OX1R in the BLA mimicked the effects of systemic OX1R antagonist administration, further supporting the relevance of OX1R signalling within this brain structure during contextual fear extinction.

Several studies measuring immediate-early gene expression conclude that hypoactivity of specific brain areas of the corticoamygdala circuit, such as the infralimbic medial prefrontal cortex (IL) and the BLA, is related to impaired fear extinction (Holmes and Singewald, 2013). Furthermore, complete extinction of conditioned fear is associated with increased expression of markers for neuronal activation in these brain areas (Herry and Mons, 2004). Distinct populations of BLA neurons encode fear conditioning and extinction processes (Herry et al., 2008). These cell populations, functionally defined as "fear neurons" and "extinction neurons," overlap with 2 different anatomically defined subtypes of BLA neurons projecting to prelimbic medial prefrontal cortex (PL) or IL, respectively (Senn et al., 2014). Nonpyramidal GABAergic interneurons within the BLA can be classified by the content of several calcium-binding proteins such as calbindin (CB) and/or parvalbumin (PV) (McDonald and Mascagni, 2001; Capogna, 2014), which could differentially control these neuronal circuits since they are involved in fear and anxiety (Butler et al., 2011; Wolff et al., 2014). Whether orexins affect these circuits and GABAergic interneuron subpopulations during emotional memory processes remain unexplored.

In this study, we used retrograde tracing and immunofluorescence techniques to identify cell subtypes within the BLA influenced by orexin antagonism during the process of contextual fear extinction.

\section{Methods}

\section{Animals}

Male C57BL6/J mice (12 weeks old, Janvier) were individually housed for 7 days prior to experiments and provided with food and water ad libitum. Housing was maintained at constant temperature $\left(21 \pm 1^{\circ} \mathrm{C}\right)$ and humidity $(55 \pm 10 \%)$ under a 12-h-light/-dark cycle, and all studies were performed during the light phase. All animal procedures were conducted in accordance with standard ethical guidelines (European Communities Directive 86/60-EEC) and were approved by the committee on Animal Health and Care of Institut National de la Santé et de la Recherche Médicale and French Ministry of Agriculture and Forestry (agreement A3312001).

\section{Drugs}

The OX1R antagonist SB334867 (5 mg/kg) (Tocris) was dissolved

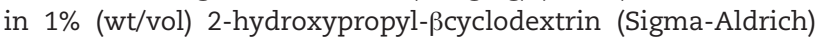
and $10 \%$ dimethyl-sulfoxide (vol/vol) in distilled water, and administered by i.p. route $(5 \mathrm{~mL} / \mathrm{kg})$.

\section{Contextual Fear Extinction}

Mice were fear conditioned in a shuttle chamber surrounded by a larger sound-attenuating cabinet (Imetronic). The shuttle chamber floor was constructed of parallel stainless-steel bars and connected to a scrambled shock generator. On the training day, mice were placed in the conditioning chamber for 3 minutes before the exposure to an unconditioned stimulus (US, 0.6-mA footshock for
$2 \mathrm{~s})$ and then remained in the chamber for 1 minute. Testing was performed during 2 extinction trials (E1 and E2 at 24 and 48 hours after the training, respectively) by scoring freezing behavior during the first 3 minutes of a 5-minute exposure to the conditioning chamber. Freezing, a rodent's natural response to fear (LeDoux, 1993), was defined as the absence of movement and was scored by an automated infrared beam detection system located on the bottom of the experimental chambers (Imetronic). SB334867 or vehicle was administered immediately after the first extinction session (E1), targeting the consolidation phase of fear extinction.

\section{Stereotaxic Surgery and Retrograde Labeling}

We used fluorophore-coated latex microspheres (red excitation $=530 \mathrm{~nm}$, emission $=590 \mathrm{~nm}$; and green excitation $=460 \mathrm{~nm}$, emission $=505 \mathrm{~nm}$ ) referred to as retrobeads (Lumafluor) for retrograde labeling of BLA neurons projecting to the PL or IL. Mice were anesthetized with isoflurane (induction 3\%, maintenance $1.5 \%$ ) in oxygen. Body temperature was maintained at $37^{\circ} \mathrm{C}$ with a temperature controller system. Mice were secured in a stereotaxic frame and injected unilaterally with differently colored retrobeads to the PL and IL using a syringe in combination with glass pipettes (borosilicate glass capillaries pulled on a micropipette puller P-97, Sutter Instruments). One-half of the animals were injected with red retrobeads in the PL and green in the IL, and the other one-half with the inverse combination, to avoid bead-induced bias in visualization and/or retrotracing. The injection volume for each region of the medial prefrontal cortex (mPFC) was $0.2 \mu \mathrm{L}$. Coordinates to target PL were: anteroposterior $+1.80 \mathrm{~mm}$, mediolateral $\pm 0.25 \mathrm{~mm}$, and dorsoventral $-1.60 \mathrm{~mm}$ relative to bregma; IL was targeted in a $16^{\circ}$ angle at: anteroposterior $+1.80 \mathrm{~mm}$, mediolateral $\pm 0.95 \mathrm{~mm}$, and dorsoventral $-2.70 \mathrm{~mm}$ relative to bregma. Injection micropipette was removed from the brain 20 minutes after each injection to minimize tracing outside the injection site. Mice were individually housed and allowed to recover for 4 weeks before behavioral training in order to grant substantial retrograde bead transport.

\section{Immunofluorescence and Imaging}

\section{Tissue Preparation}

Two hours after the second extinction trial (E2), retrobead-injected mice were transcardially perfused with $4 \%$ paraformaldehyde, postfixed in the same fixative for 24 hours $\left(4^{\circ} \mathrm{C}\right)$, and cryoprotected in a solution of $30 \%$ sucrose at $4^{\circ} \mathrm{C}$. Coronal frozen sections containing the BLA (from $-1.22 \mathrm{~mm}$ to $-1.82 \mathrm{~mm}$ relative to bregma) and the $\mathrm{mPFC}$ (from $+1.98 \mathrm{~mm}$ to $+1.54 \mathrm{~mm}$ relative to bregma) were made at $30 \mu \mathrm{m}$ on a freezing microtome and stored in a $5 \%$ sucrose solution at $4^{\circ} \mathrm{C}$ until use. Slices containing the caudal and middle BLA regions were selected for immunofluorescence processing.

\section{Injection Site Verification}

Serial mPFC coronal sections were mounted onto glass slides. Low-magnification images from all slices showing retrobead presence were obtained using a Zeiss fluorescent microscope, and retrobead injection sites were histologically verified by overlapping to standard stereotaxic plates (Paxinos and Franklin, 2001). Only animals with retrobead presence circumscribed into the PL or the IL were included in statistical analysis of c-Fos expression within retrolabeled neurons (12 of 23 mice).

\section{Immunofluorescence}

Free-floating BLA slices were rinsed in 0.1 M PB, blocked in a solution containing $3 \%$ normal goat serum and $0.3 \%$ Triton $\mathrm{X}-100$ in 
0.1M PB (NGS-T-PB) at room temperature for 2 hours, and incubated overnight at $4^{\circ} \mathrm{C}$ in the same solution with the primary antibody. Next day, after 3 rinses in $0.1 \mathrm{M} \mathrm{PB}$, sections were incubated with the secondary antibody at room temperature in NGS-T-PB for 2 hours. After incubation, sections were rinsed and mounted immediately after onto glass slides coated with gelatine in Mowiol mounting medium. We used the following primary antibodies: anti-c-Fos (1:500, rabbit, sc-72, Santa Cruz Biotechnology), anti-PV (1:500, guinea pig, Synaptic Systems), and anti-CB (1:500, mouse, Synaptic Systems). As secondary antibodies, we employed AlexaFluor-647 goat anti-rabbit (1:500, Life Technologies), AlexaFluor-555 goat anti-guinea pig (1:500, ThermoFisher), and AlexaFluor-488 goat anti-mouse (1:500, Jackson ImmunoResearch).

\section{Image Analysis}

Confocal images were obtained using a Leica TCS SP5 confocal microscope (20x objective) (Leica Microsystems). Threedimensional $\mathrm{z}$ stacks were acquired using 3 different laser lines $(488,561$, and $633 \mathrm{~nm})$, sectioned in $4-\mu \mathrm{m}$ thin optical planes along 16 to $20 \mu \mathrm{m}$ (5-6 planes). Two-dimensional overview pictures (8 bit, $1024 \times 1024$ pixels) were obtained by $\mathrm{z}$ projection and further analyzed in ImageJ software (6 sections per animal). Expression of c-Fos was determined bilaterally within the BLA using a fixed threshold interval ( $\mathrm{n}=11-12$ mice/group). Colocalization of c-Fos with red or green retrobead positive neurons ( $n=6$ mice/group), as well as with PV or CB positive neurons ( $n=9$ mice/group), was quantified using the ImageJ manual particle counting option. PV and $\mathrm{CB}$ were analyzed unilaterally to avoid retrobead detection.

\section{Statistical Analysis}

Repeated-measures 2-way ANOVA was employed to analyze freezing behavior, followed by Fisher LSD posthoc test. Unpaired Student's t test was used in histological experiments to compare vehicle- and SB334867-treated groups. The level of significance was $P<0.05$ in all experiments.

\section{Results}

\section{Treatment with the OX1R Antagonist SB334867 Is Associated with Increased Activity of BLA Neurons Projecting to IL during Fear Extinction}

Mice were injected unilaterally into the PL and IL with 2 different retrobeads coated with red or green fluorophores (Figure $1 \mathrm{~A}$ and E) to assess whether orexin antagonism modifies the activation pattern of the putative fear (PL-projecting) and extinction (IL-projecting) neurons present in the BLA (Senn et al., 2014) during contextual fear extinction. After 4 weeks of recovery to allow sufficient axonal retrobead transport, mice underwent a contextual fear conditioning and extinction procedure. Freezing behavior was scored during the first extinction session (E1), mice were treated with SB334867 (5 mg/kg, i.p.) or vehicle immediately after, and $24 \mathrm{~h}$ later (second extinction session, E2) freezing was measured again (Figure 1A). As previously reported (Flores et al., 2014), OX1R antagonism was able to modify the course of contextual fear extinction as shown by 2-way ANOVA (treatment $\mathrm{x}$ day: $\left.F_{(1,21)}=5.90 ; P<.05\right)$. Thus, SB334867-treated mice showed reduced levels of freezing at E2 compared with the vehicle group $(P<.05)$ (Figure $1 B)$, confirming the enhanced consolidation of fear extinction due to OX1R blockade (Flores et al., 2014). Immunofluorescence processing of the BLA after E2 revealed an increase of $\mathrm{C}$-Fos expression in SB334867-treated mice $(P<.01)$ (Figure 1C-D). Successful retrograde labeling was then confirmed by red and green retrobead detection in different projection neurons located within the BLA (Figure 1F), which resulted similarly in SB334867- and vehicle-treated groups (Figure 1G). Notably, analysis of retrobead and c-Fos coexpression showed an increased activity of BLA neurons targeting the IL $(P<.01)$ (Figure $1 F)$ in mice injected with SB334867, suggesting that OX1R blockade facilitates the recruitment of these extinction-promoting projection neurons. In contrast, no significant changes between groups were observed in the activity of PL-projecting neurons (Figure 1F). In addition, the number of nonretrolabeled c-Fos positive neurons in the BLA was higher in the SB334867 group than in the vehicle-treated group $(P<.01)$ (Figure $1 \mathrm{H})$, suggesting that the OX1R antagonist is enhancing as well the activation of other neurons than those projecting to the IL (e.g., local interneurons). The total number of retrobead+, c-Fos+, and double-positive cells of each experimental condition is shown in supplementary Table 1.

\section{OX1R Blockade Enhances the Activation of Local Interneurons Containing $C B$, but Not PV, within the BLA during Fear Extinction}

To identify which other BLA neuronal populations are activated upon OX1R blockade during fear extinction, we investigated the 2 most abundant groups of local interneurons within this brain area: those containing the calcium-binding proteins PV and CB. These cell subpopulations are mainly nonpyramidal GABAergic interneurons (McDonald and Mascagni, 2001) and are involved in fear and anxiety processes (Butler et al., 2011; Wolff et al., 2014). In agreement with previous studies, approximately $80 \%$ of PV neurons exhibited colocalization with $\mathrm{CB}$, and the number of neurons expressing $\mathrm{PV}$ and/or $\mathrm{CB}$ was not modified in mice treated with the OX1R antagonist (Figure 2A). Interestingly, mice treated with SB334867 displayed higher expression of c-Fos within those nonpyramidal neurons containing $\mathrm{CB}$ only compared with control animals $(P<.01)$ (Figure $2 B)$. This effect was not observed in either the neuronal population expressing PV or both PV and CB (Figure 2B). The total number of $\mathrm{CB}+, \mathrm{PV}+$, and c-Fos+ cells, and double-/triple-stained cells for each experimental condition is shown in supplementary Table 2. Other projection cells or inhibitory interneurons within the BLA could likewise be activated due to orexin antagonism during fear extinction, since c-Fos expression also increased in neurons that do not express CB or PV in SB334867-treated mice $(P<.01)$ (supplementary Table 2).

\section{Discussion}

Our results identify specific neuronal subpopulations within the BLA associated with enhanced consolidation of fear extinction induced by OX1R blockade. Thus, the OX1R antagonist SB334867 increased the recruitment of BLA projection neurons targeting the IL during the ensuing fear extinction trial. Moreover, GABAergic interneurons related to fear modulation that express the calcium-binding protein $\mathrm{CB}$, but not PV, were activated by OX1R antagonism during this process.

Several recent reports have revealed a role for the orexin system in the regulation of emotional memories (Flores et al., 2015). OX1R blockade facilitates the consolidation of fear extinction (Flores et al., 2014), suggesting a potential usefulness of OX1R antagonists for the treatment of anxiety disorders associated with fear deregulation, such as phobias or posttraumatic stress disorder. In agreement, the activation level of hypothalamic orexin neurons appears to be negatively correlated with successful extinction of cue-conditioned fear in rodents (Sharko et al., 2016). The BLA is a pivotal brain region 
A

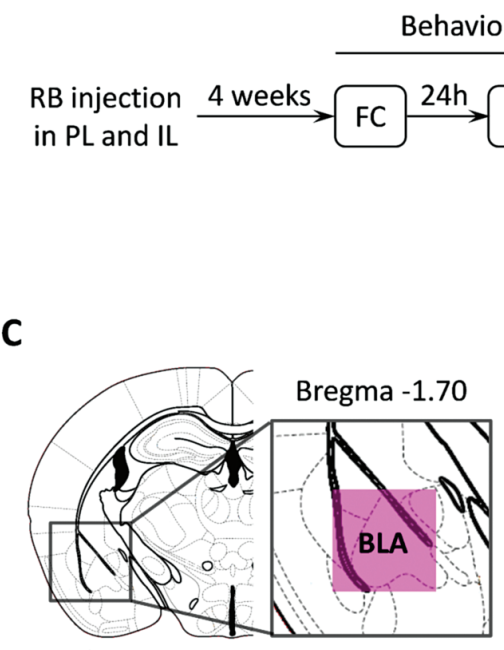

B

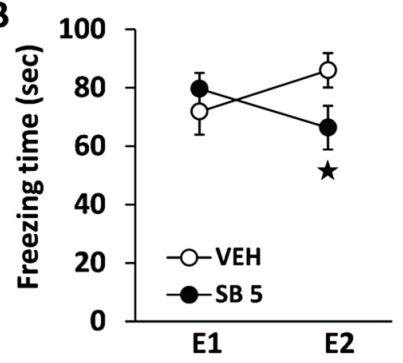

D

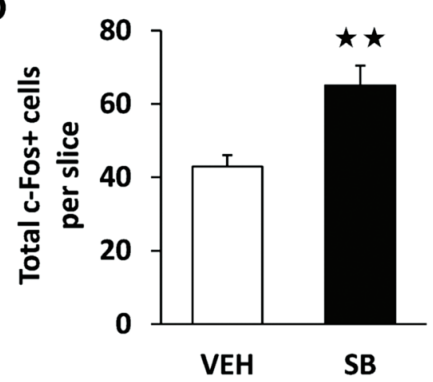

Histological analysis of BLA

VEH or SB

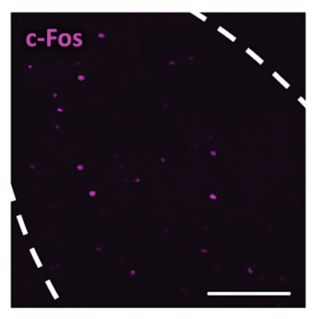

VEH

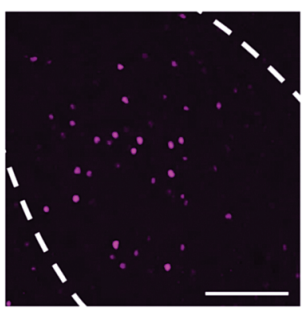

SB
E

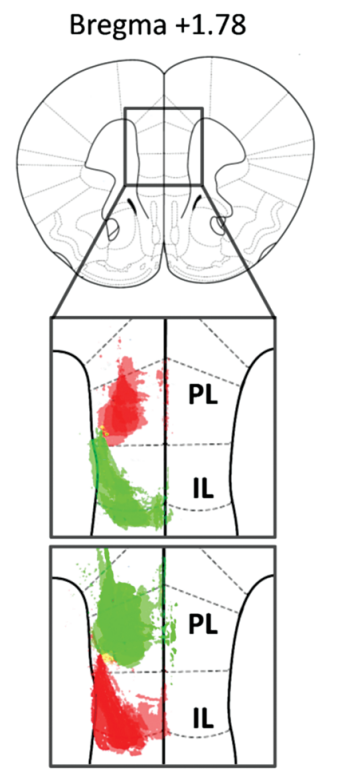

F
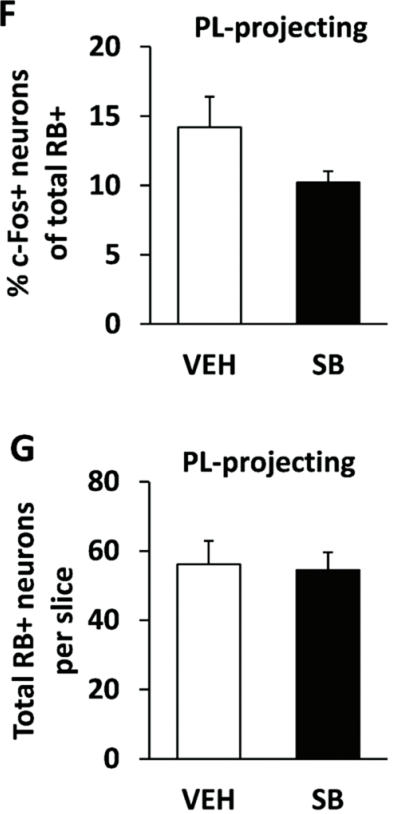

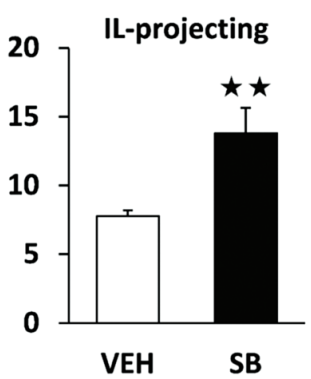

IL-projecting

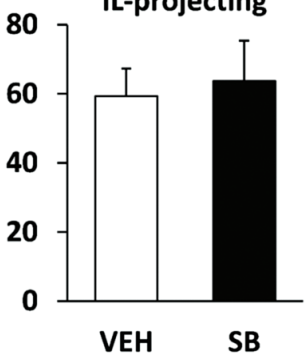

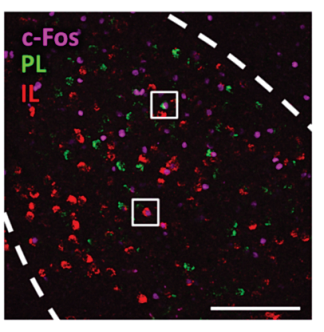

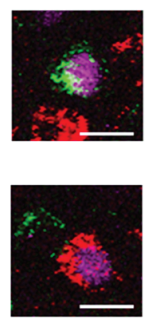

H

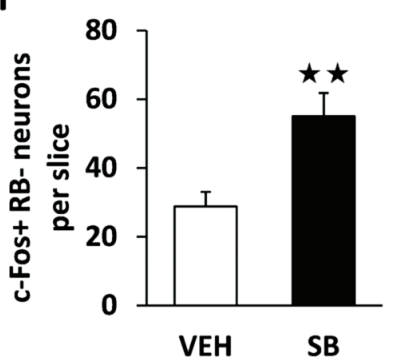

Figure 1. OX1R antagonism is associated with increased activity of basolateral amygdala (BLA) neurons projecting to infralimbic medial prefrontal cortex (IL) during fear extinction. A, Experimental design. Animals were injected with differently colored retrobeads into the prelimbic medial prefrontal cortex (PL) and IL. After 4 weeks of recovery to allow successful retrograde labelling, mice underwent contextual fear conditioning (FC), and freezing behavior was scored 24 hours later during contextual reexposure (extinction session 1, E1). Immediately after E1, mice were treated with the OX1R antagonist SB334867 (SB, 5 mg/kg, i.p.) or vehicle (VEH), and freezing behavior was measured again 24 hours later (extinction session 2, E2). Animals were sacrificed for histochemical analysis 2 hours after E2. B, Time spent freezing by VEH- and SB-treated mice during E1 and E2 ( $\mathrm{n}=12-13$ mice/group). C, Diagram showing anatomic location of BLA analyzed images. D, Number of c-Fos+ neurons within the BLA in VEH- and SB-treated mice after the E2 session ( $\mathrm{n}=12-13$ mice/group; left panel). Representative images of c-Fos immunofluorescence (purple) in the BLA obtained by confocal microscopy are also shown (right panel). E, Diagram displaying injection sites within the medial prefrontal cortex (mPFC) of those mice suitable for histochemical analysis: upper panel shows overlaid fluorescence detected in 3 VEH- and 2 SB-treated mice, whereas lower panel corresponds to 3 VEH- and 4 SB-treated mice. F, Percentage of PL- and IL-projecting neurons per slice expressing c-Fos in mice receiving VEH or SB (left panel; $\mathrm{n}=6 \mathrm{mice} / \mathrm{group}$ ). Representative images of c-Fos (purple) and retrobead (red and green) colocalization at low and high magnification are also shown (right panel). G, Total number of retrolabelled neurons per slice projecting to PL and IL ( $n=6$ mice/group). H, Number of c-Fos+ nonretrolabelled neurons per slice ( $n=6$ mice/group). RB, retrobeads. Scale bars represent $200 \mu \mathrm{m}$ in whole BLA pictures and $20 \mu \mathrm{m}$ in high magnification pictures. ${ }^{*} \mathrm{P}<.05,{ }^{* *} \mathrm{P}<.01$ between treatments.

for processing fear, and decreased activity of this structure has been linked to impairment of fear extinction (Holmes and Singewald, 2013). Accordingly, the extinction-facilitating effects of the OX1R antagonist SB334867 were associated with increased c-Fos expression in the BLA, as previously reported (Flores et al., 2014).
The BLA and the mPFC are interconnected brain structures involved in the acquisition and extinction of conditioned fear (Dejean et al., 2015). The IL subdivision of the mPFC plays a crucial role in extinction memory consolidation, while the adjacent PL subdivision has been implicated in sustained fear expression and resistance to extinction (Sierra-Mercado et al., 2011). 

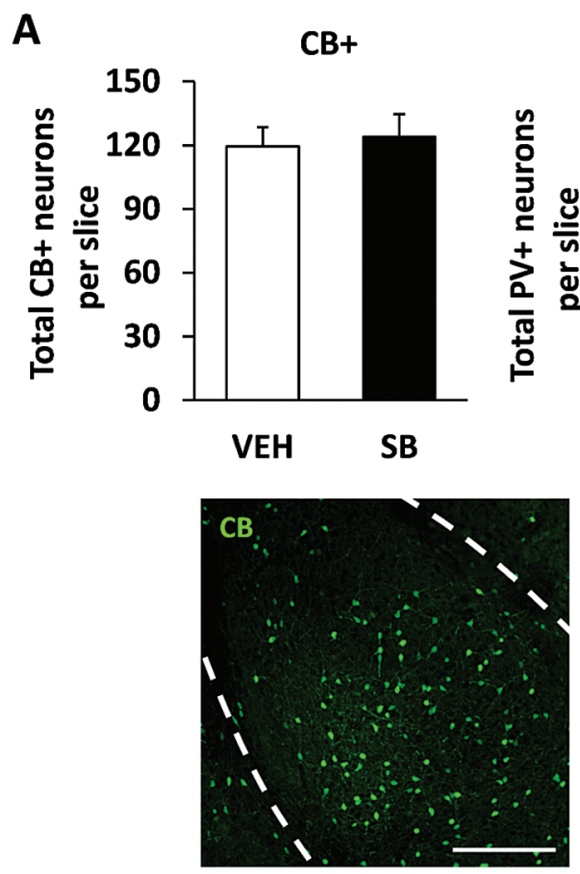
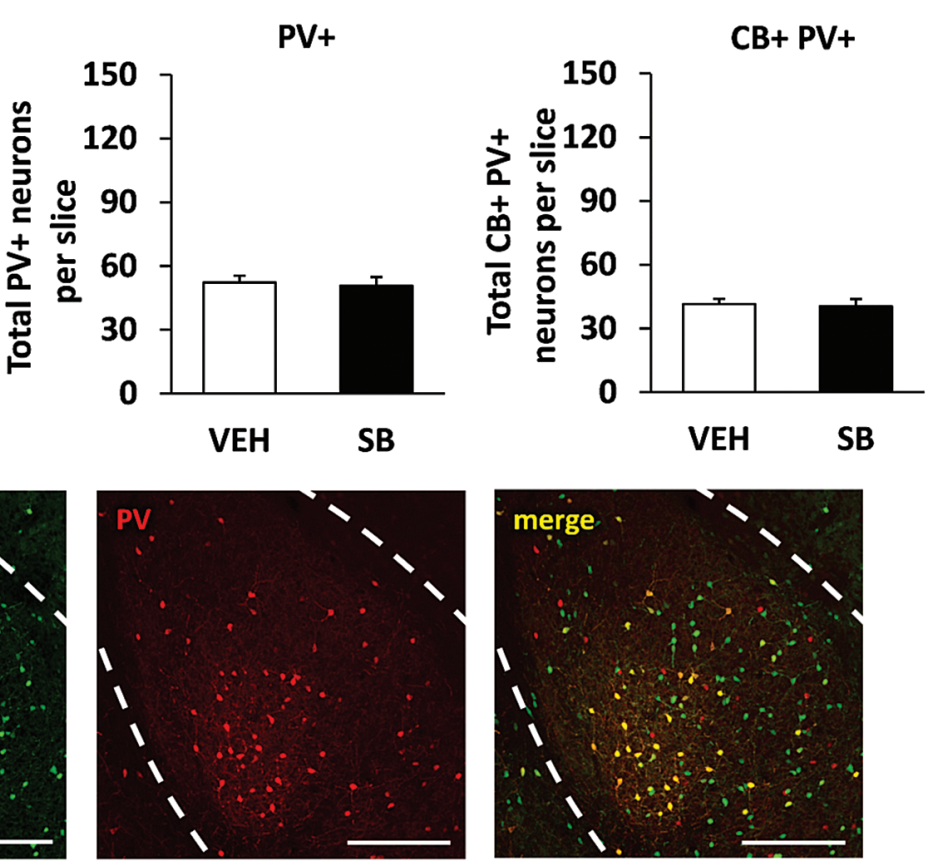

B
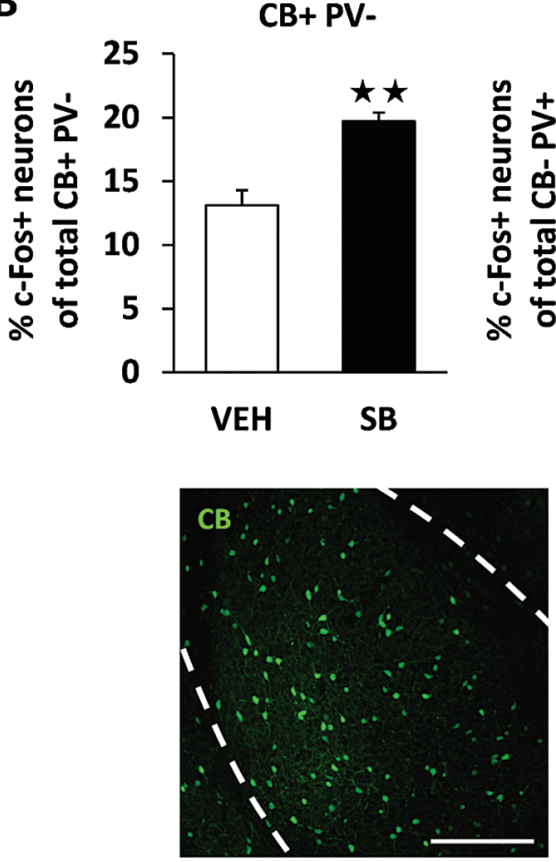

CB- PV+
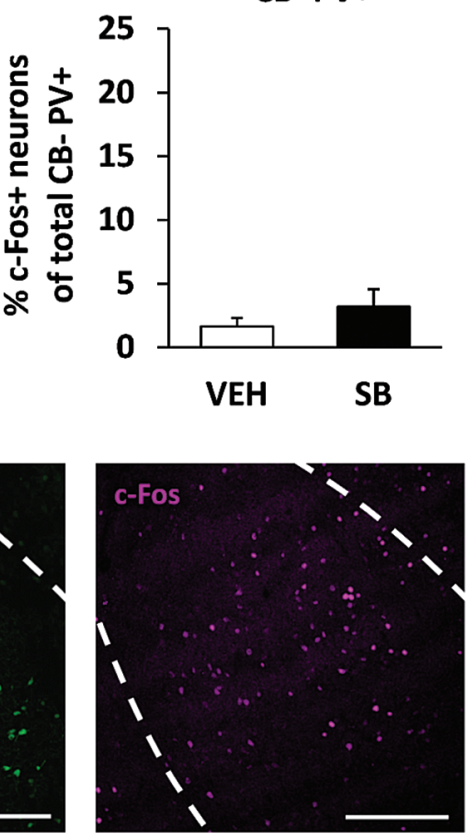
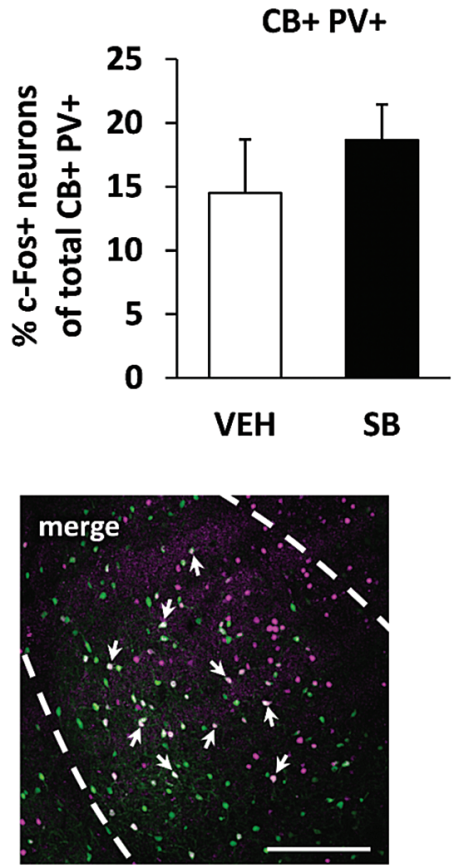

Figure 2. OX1R antagonism increases c-Fos expression in calbindin (CB)+ neurons within the basolateral amygdala (BLA) during fear extinction. A, Total number of CB+ neurons, parvalbumin (PV)+ neurons, and coexpressing $\mathrm{CB}+\mathrm{PV}+$ neurons per slice detected within the BLA ( $\mathrm{n}=9$ mice/group). Representative images showing $\mathrm{CB}+$ (green), PV+ (red), and CB+PV+ (yellow) immunofluorescence is also displayed (lower panels). B, Percentage of c-Fos+ neurons among those expressing only CB, only PV, or coexpressing CB and PV. Representative images showing c-Fos (purple) expression within CB+ (green) neurons is also displayed (lower panels). Arrows indicate CB+ neurons expressing c-Fos. Scale bars represent $200 \mu \mathrm{m} .{ }^{* *} \mathrm{P}<.01$ between treatments.

Recently, an elegant study using retrograde tracing and optogenetic approaches has revealed the existence of distinct subpopulations of BLA projection neurons to the MPFC in fear expression and extinction (Senn et al., 2014). BLA neurons projecting to PL are active during high states of fear, whereas BLA neurons targeting the IL are recruited during fear extinction (Senn et al., 2014). Notably, we observed an enhancement in the activation of
BLA neurons projecting to IL, but not to PL, in mice treated with SB334867 during fear extinction. In agreement, OX1R antagonism has been reported to increase IL activation during this behavioral response (Flores et al., 2014). These results suggest that the facilitation of fear extinction consolidation induced by OX1R antagonism is associated with the recruitment of this BLA-IL neuronal circuit. 
Nonpyramidal GABAergic interneurons within the BLA can be divided considering the content of several calcium-binding proteins such as CB and/or PV (McDonald and Mascagni, 2001). These cell subtypes have been previously involved in the regulation of fear behaviors (Butler et al., 2011; Wolff et al., 2014) and could differentially modulate neuronal circuits related to fear conditioning and extinction processes. The administration of SB334867 increased the activation of neurons containing CB, but not PV, during the extinction of fear. In agreement, activation of CB-expressing interneurons appears to be inversely correlated with fear expression (Butler et al., 2014). Thus, rats exposed to predator odor displayed increased fear responses and reduced c-Fos expression in CB+ neurons in the BLA (Butler et al., 2011). PV neurons were not affected by the exposition to the same predator threat (Butler et al., 2011). Together, these data suggest that declined fear expression during the extinction process induced by OX1R antagonism could be due to the activation of local BLA interneurons expressing CB. Nevertheless, intraamygdalar microcircuits are still poorly understood, and hence it remains to be elucidated whether this increased activity of CB-expressing interneurons by OX1R blockade is related to the modulation of the BLA-IL circuit.

In conclusion, these data enhance our knowledge of the amygdala circuitry involved in the facilitation of fear extinction induced by OX1R antagonism. Such understanding will contribute to refine future therapeutic approaches for anxiety disorders characterized by pathological fear.

\section{Acknowledgments}

This work was supported by "Plan Nacional sobre Drogas" (no. 2014I019 to F.B.), the "Ministerio de Economía y CompetitividadMINECO" (no. SAF2014-59648-P to R.M.), the "Instituto de Salud Carlos III" (no. PI13/00042 to F.B. and RETICS-RTA, no. RD12/0028/0023 to R.M.), the "Generalitat de Catalunya-AGAUR" (no. 2014-SGR-1547 and ICREA-Acadèmia (2015) to R.M.).

AF was recipient of an EMBO Short-Term Fellowship (281-2015).

\section{Statement of Interest}

None.

\section{References}

Butler RK, Sharko AC, Oliver EM, Brito-Vargas P, Kaigler KF, Fadel JR, Wilson MA (2011) Activation of phenotypically-distinct neuronal subpopulations of the rat amygdala following exposure to predator odor. Neuroscience 175:133-144.

Capogna M (2014) GABAergic cell type diversity in the basolateral amygdala. Curr Opin Neurobiol 26:110-116.

Dejean C, Courtin J, Rozeske RR, Bonnet MC, Dousset V, Michelet T, Herry C (2015) Neuronal circuits for fear expression and recovery: recent advances and potential therapeutic strategies. Biol Psychiatry 78:298-306.

de Lecea L, Kilduff TS, Peyron C, Gao X, Foye PE, Danielson PE, Fukuhara C, Battenberg EL, Gautvik VT, Bartlett FS 2nd, Frankel WN, van den Pol AN, Bloom FE, Gautvik KM, Sutcliffe JG (1998) The hypocretins: hypothalamus-specific peptides with neuroexcitatory activity. Proc Natl Acad Sci U S A 95:322-327.

Flores Á, Valls-Comamala V, Costa G, Saravia R, Maldonado R, Berrendero $F$ (2014) The hypocretin/orexin system mediates the extinction of fear memories. Neuropsychopharmacology 39:2732-2741.
Flores Á, Saravia R, Maldonado R, Berrendero F (2015) Orexins and fear: implications for the treatment of anxiety disorders. Trends Neurosci 38:550-559.

Herry C, Ciocchi S, Senn V, Demmou L, Müller C, Lüthi A (2008) Switching on and off fear by distinct neuronal circuits. Nature 454:600-606.

Herry C, Mons N (2004) Resistance to extinction is associated with impaired immediate early gene induction in medial prefrontal cortex and amygdala. Eur J Neurosci 20:781-790.

Holmes A, Singewald N (2013) Individual differences in recovery from traumatic fear. Trends Neurosci 36:23-31.

LeDoux JE (1993) Emotional memory: in search of systems and synapses. Ann NY Acad Sci 702:149-157.

Li J, Hu Z, de Lecea L (2014) The hypocretins/orexins: integrators of multiple physiological functions. Br J Pharmacol 171:332-350.

McDonald AJ, Mascagni F (2001) Colocalization of calciumbinding proteins and GABA in neurons of the rat basolateral amygdala. Neuroscience 105:681-693.

Paxinos G, Franklin KB. 2001. The mouse brain in stereotaxic coordinates. San Diego: Academic Press.

Peyron C, Tighe DK, van den Pol AN, de Lecea L, Heller HC, Sutcliffe JG, Kilduff TS (1998) Neurons containing hypocretin (orexin) project to multiple neuronal systems. J Neurosci 18:9996-10015.

Peyron C, Faraco J, Rogers W, Ripley B, Overeem S, Charnay Y, Nevsimalova S, Aldrich M, Reynolds D, Albin R, Li R, Hungs M, Pedrazzoli M, Padigaru M, Kucherlapati M, Fan J, Maki R, Lammers GJ, Bouras C, Kucherlapati R, Nishino S, Mignot E (2000). A mutation in a case of early onset narcolepsy and a generalized absence of hypocretin peptides in human narcoleptic brains. Nat Med 6:991-997.

Ponz A, Khatami R, Poryazova R, Werth E, Boesiger P, Schwartz S, Bassetti CL (2010) Reduced amygdala activity during aversive conditioning in human narcolepsy. Ann Neurol 67:394-398.

Sakurai T, Amemiya A, Ishii M, Matsuzaki I, Chemelli RM, Tanaka H, Williams SC, Richardson JA, Kozlowski GP, Wilson S, Arch JR, Buckingham RE, Haynes AC, Carr SA, Annan RS, McNulty DE, Liu WS, Terrett JA, Elshourbagy NA, Bergsma DJ, Yanagisawa M (1998) Orexins and orexin receptors: a family of hypothalamic neuropeptides and G protein-coupled receptors that regulate feeding behavior. Cell 92:573-585.

Sears RM, Fink AE, Wigestrand MB, Farb CR, de Lecea L, Ledoux JE (2013) Orexin/hypocretin system modulates amygdaladependent threat learning through the locus coeruleus. Proc Natl Acad Sci U S A 110:20260-20265.

Senn V, Wolff SB, Herry C, Grenier F, Ehrlich I, Gründemann J, Fadok JP, Müller C, Letzkus JJ, Lüthi A (2014) Long-range connectivity defines behavioral specificity of amygdala neurons. Neuron 81:428-437.

Sharko AC, Fadel JR, Kaigler KF, Wilson MA (2016) Activation of orexin/hypocretin neurons is associated with individual differences in cued fear extinction. Physiol Behav (in press).

Sierra-Mercado D, Padilla-Coreano N, Quirk GJ (2011) Dissociable roles of prelimbic and infralimbic cortices, ventral hippocampus, and basolateral amygdala in the expression and extinction of conditioned fear. Neuropsychopharmacology 36:529-538.

Soya S, Shoji H, Hasegawa E, Hondo M, Miyakawa T, Yanagisawa M, Mieda M, Sakurai T (2013) Orexin receptor-1 in the locus coeruleus plays an important role in cue-dependent fear memory consolidation. J Neurosci 33:14549-14557.

Wang H, Li S, Kirouac GJ (2017) Role of the orexin (hypocretin) system in contextual fear conditioning in rats. Behav Brain Res 316:47-53.

Wolff SB, Gründemann J, Tovote P, Krabbe S, Jacobson GA, Müller C, Herry C, Ehrlich I, Friedrich RW, Letzkus JJ, Lüthi A (2014) Amygdala interneuron subtypes control fear learning through disinhibition. Nature 509:453-458. 